\title{
Fast-track Cardiac Surgery in Children: Feasibility in Bangladeshi Setting
}

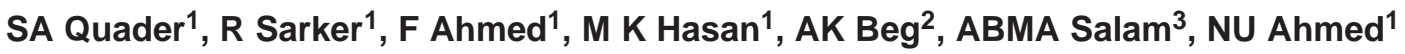 \\ ${ }^{1}$ Department of Cardiac Surgery, NICVD, ${ }^{2}$ Department of Anesthesia, NICVD, ${ }^{3}$ Department of \\ Paediatric Cardiology, NICVD.
}

Keywords:
Cardiac surgery,
Congenital heart
disease,
Fast track,
Hospital
discharge.

\begin{abstract}
:
Background: Fast-track recovery protocols in cardiac surgery is gaining worldwide popularity and have contributed to significant reductions in the postoperative hospital stay and cost without any increase in postoperative mortality and morbidity. The aim of this study was to find out the feasibility of fast track paediatric cardiac surgery in Bangladeshi setting.

Method: It was a prospective study conducted in National Institute of Cardio-vascular Diseases, Dhaka, from July 2009 to June 2010. All patients, between 3 to 18 years, underwent surgical closure of atrial or ventricular septal defect under cardio-pulmonary bypass. 20 patients from our unit served as fast track group and 30 patients from other units of the same hospital served as conventional group. Fast track patients were extubated in less than 6 hours after surgery, shifted from ICU in less than 24 hours and geared up to discharge home within 3 days of surgery.
\end{abstract}

Result: 18 (90\%) of the 20 patient of the fast track group were discharged within 3 days of surgery, 2 patients cannot be discharged within this time frame. Mean post operative hospital stay for study group was 3.1 days, whereas the mean hospital stay in the control group was 7.5 days. Follow-up was $100 \%$ complete at 30 days. There was no major in-hospital or out-of-hospital complications in either group. No patient was readmitted at our centre or elsewhere for any complication arising from this process.

Conclusion: Fast tracking is feasible and safe in low-risk paediatric open-heart surgery in Bangladeshi scenario. A multidisciplinary approach with a set protocol is required to achieve this goal in a safe and reproducible manner.

(Cardiovasc. j. 2010; 3(1) : 50-54)

\section{Introduction:}

The term fast-track surgery was established by the Danish surgeon, Helmut Kehlet, ${ }^{1,2}$ in the 1990s and represents a comprehensive program for optimization of perioperative care in elective surgery, by reducing stress and discomfort and speeding up convalescence. Conventional management after open heart surgery includes a period of elective ventilation coupled with sedation and paralyzing agents, a prolonged period of recovery and delayed return of the patient to normal activity. Improvements in cardiopulmonary bypass (CPB) techniques, specifically advances in membrane oxygenation and blood sparing techniques have reduced the cerebral, pulmonary, renal and bleeding complications of cardiac surgery. ${ }^{3,4}$ Shorter CPB times, improved myocardial protection and new anaesthetic methods allow extubation within 6 hours postoperatively, assuming haemodynamic stability. ${ }^{5-7}$ Such patients can then be discharged to a 'stepdown' or high dependency unit (HDU). As a result, fast-track recovery protocols gained worldwide popularity and have contributed to significant reductions in the postoperative hospital stay and cost without any increase in postoperative mortality and morbidity. ${ }^{8-11}$ In Bangladesh, on the other hand, patients stay in hospital much longer than in developed countries because of lack of a set protocol and good understanding between the members of cardiac surgical team about fasttracking the patients. There is also lack of facilities for regular follow up of the patient outside the operating hospital which causes hesitation on the part of the operating surgeon to discharge the patient early. In some case patient wants to stay

Address of Correspondence : Dr. Sayed A. Quader, Department of Cardiac Surgery, NICVD, Dhaka, Bangladesh. e-mail:dr.saquader.ms@gmail.com 
in hospital as they are apprehensive of any complication that may arise after discharge. But we need to get used to fast-track pathway to make full use of a limited health care resource. A fast track recovery protocol has been set in our unit for about a year. The aim of this study was to determine if the protocol increases mortality and morbidity by comparing the conventional group and the fast-track recovery protocol group in regard of postoperative length of stay, morbidity and mortality.

\section{Patients and Methods:}

\section{Study population:}

It was a prospective study conducted in National Institute of Cardio-vascular Diseases, Dhaka, from July 2009 to June 2010. This prospective study was approved by the local ethical committee. All patients underwent low risk ${ }^{12}$ open-heart surgery (surgical closure of atrial or ventricular septal defect) with CPB due to congenital heart disease. Of this, 20 patients from our unit served as fast track group and 30 patients from other units of the same hospital served as conventional group. All patients in the fast track group were required to have an accompanying family member or a friend available for hospitalization time and for 72 hours thereafter and they should have accommodation to stay in Dhaka city for at least 1 week after discharge. Exclusion criteria were age $<3$ years or $>18$ years, re-operations, secondary pulmonary hypertension, severe heart failure (New York Heart Association stages III and IV), reduced left ventricular shortening fraction in cardiac ultrasound $(<35 \%)$ or other co morbidities like renal, hepatic, pulmonary disease. Once appropriateness of the patient for this approach was determined based on inclusion criteria, Informed consent was obtained from all patients who entered the study protocol. The entire process of counseling, investigations and pre-anesthetic check-up was completed before hospital admission. Patients were provided with the date of surgery and the telephone number of a senior resident to contact in case of confusion or delay/failure to arrive on the said date. All patients were admitted at least 1 day prior to surgery. A rapid-recovery protocol emphasizing reduced CPB time, an anesthesia protocol for early extubation, perioperative administration of corticosteroids and Tranexamic acid and aggressive diuresis was applied to all patients. The object during the first 24 hours postoperatively was to achieve early extubation as well as a mild state of negative fluid balance and to ensure absence of postoperative bleeding and a safe transfer from the intensive care unit to ward.

\section{Anaesthetic technique:}

A consultant anaesthetist assessed all patients for fitness for anaesthesia and surgery. The anaesthetic technique and drugs were tailored towards early extubation in case of first track group. Venous access with a large bore cannula and invasive blood pressure monitoring was established under local anaesthesia. Following preoxygenation, anaesthetic induction was achieved with 10-15 mcg/kg Fentanyl, an induction dose of propofol $100-150 \mathrm{mcg} / \mathrm{kg}$ of Pancuronium for muscle relaxation. Following tracheal intubation intermittent positive pressure ventilation (IPPV) was established. Central venous access was established with a multi-lumen catheter. Intraoperative transoesophageal echocardiography (TOE) was used if indicated. Anaesthesia was maintained with air/oxygen and isoflurane mixture and intravenous Propofol infusion until bypass and then solely with Propofol at 4-6 mg/kg per hour during and after CPB. Monitoring included lead II ECG, invasive blood pressure, central venous pressure, pulse oximetry, end-tidal $\mathrm{CO} 2$, nasopharyngeal temperature, urine output, ACT and intermittent blood gas analysis in all patients.

In the control group, anaesthesia was induced with Fentanyl 8-10 mg/kg and Thiopentone 50-100 mg. Muscle relaxation was achieved with Pancuronium bromide $0.15 \mathrm{mg} / \mathrm{kg}$. Anaesthesia was maintained with hourly bolus doses of Morphin, Fentanyl, Midazolam, Pancuronium bromide and Nitrous oxide/Halothane mixed with air/oxygen.

\section{Cardiopulmonary bypass technique:}

All procedures were performed on complete CPB with standard bicaval venous and ascending aortic cannulation, membrane oxygenator, with nonpulsatile arterial flow. The priming solution contained mainly acetated Ringer's solution, and whole blood, as needed. CPB was performed under conditions of moderate hemodilution, maintaining the hematocrit $35-40 \%$, and whole body 
anticoagulation with heparin. CPB was performed with mild hypothermia (nasopharyngeal temperature- $32-34^{\circ} \mathrm{C}$ ). Perfusion pressure was maintained between 40 and $60 \mathrm{mmHg}$. Active rewarming was started while approaching termination of CPB.

\section{Surgical procedure:}

All patients underwent surgical closure of atrial septal defect (ASD) or ventricular septal defect (VSD) through median sternotomy. Meticulous care was taken to achieve adequate heamostasis to avoid postoperative bleeding and possible re exploration.

\section{Postoperative care:}

Soon after cardiac surgery, all patients were transferred to the intensive care unit (ICU). Patients were extubated 1-6 hours after their arrival in the ICU. The criteria for extubation were (1) a conscious patient obeying verbal commands, (2) adequate ventilation (oxygen saturation $>98 \%$ at a $\mathrm{FiO} 2$ of 0.40 , (3) hemodynamic stability and had a $<10$ inotropic score, ${ }^{13}$ [dose of dopamine + dobutamin + $100 \mathrm{X}$ adrinalin $(\mu \mathrm{g} / \mathrm{kg} / \mathrm{min})]$ (4) normothermia, (5) complete reversal of the neuromuscular blockade, and (6) an adequate homeostasis. If rhythm was stable with satisfactory heart rate, temporary pacing wires were removed on the morning of $1^{\text {st }}$ post operative day (POD), followed by removal of chest drains if there was no significant drainage. Majority of the patients were shifted to the ward in less than 24 hours after surgery. Any patient who required ongoing respiratory assistance, cardiovascular support drugs, fluid boluses or close monitoring with one-to-one level nursing was considered unfit for fast-track and kept in the ICU. In ward aggressive ambulation therapy was instituted. In-hospital care included routine antibiotics, analgesics, low dose diuretics and GI prophylaxis.

\section{Criteria for discharge:}

If at 72 hours postoperatively the patient was walking without assistance, had return of normal bowel function, with well controlled pain and willingness to go home a 3-day discharge home was planned. On the other hand conventional group follows a more leisurely cruse, they were shifted from ICU to a HDU on $2^{\text {nd }}$ POD where they remain for another 1 to 2 days and then shifted to ward. They usually discharged from the word on 7 to 10 PODs.

\section{Follow-up:}

Patients were followed up over telephone by a senior resident at $12 \mathrm{~h}, 24 \mathrm{~h}$ and 3 days after discharge. Clinical out-patient follow-up was performed at 7 and 30 days after discharge.

\section{Statistical analysis}

Results are expressed as mean (standard deviation) or percentage, as appropriate. Analysis between groups was conducted with the unpaired t-test. Nonparametric data was analyzed with the Ç2 test. Differences were considered significant if $\mathrm{p}<0.05$.

\section{Results:}

\section{Table-I}

Demographic and Clinical Variables

\begin{tabular}{lccc}
\hline Characteristics & Study group & Control group & P value \\
\hline Age (year) & $* 9.25 \pm 3.74$ & $* 9.12 \pm 4.22$ & 0.909 \\
Females & $11(55 \%)$ & $16(53.3 \%)$ & 1.00 \\
Body weight (kg) & $* 19.4 \pm 7.51$ & $* 19.9 \pm 8.93$ & 0.837 \\
VSD & $11(55 \%)$ & $14(46.7 \%)$ & 0.773 \\
\hline
\end{tabular}

*Mean $\pm \mathrm{SD}$

Over the 12-month period, 20 patients underwent surgical closure of ASD or VSD as fast-track patients in our unit. During the same study period, 30 patients undergoing conventional ASD or VSD closure in the other units of the department served as control group. Data from 50 patients was analyzed. The demographical data were similar for both groups (Table 1). There were no significant differences between groups in duration of surgery, CPB time and cross-clamp time. The mean time to extubation in the study group was $181( \pm 89)$ minutes This was significantly less than the control group $(p<0.002)$, which had a mean time of extubation of $354( \pm 218)$ minutes. In the fast track group 3 patients required ionotropic support for more than 12 hours and in conventional group it was 4 patients which were not statistically significant. The mean length of stay in the ICU was $23.92( \pm 8.7)$ hours for the study group and $47.29( \pm 13.7)$ hours for the control group which is statistically significant. None of the patient required re-intubation, re-exploration or readmission to ICU. Of the 20 patients in fast-track group, $18(90 \%)$ successfully shifted to word in less than 24 hours and 2 patients (10\%) stayed for more than 24 hours in ICU for cardiovascular instability requiring inotropes. 18 (90\%) patients were successfully discharged within 3 days of surgery (mean hospital stay after surgery was $68.24 \mathrm{hrs}$ ). 2 patients remained in-hospital for 7 and 4 days postoperatively because of superficial wound infection and pleural effusion. There was significant difference in postoperative hospital stay between 
Table-II

Operative and post operative variable

\begin{tabular}{lccc}
\hline Variables & Study group(n=20) & Control group(n=30) & P value \\
\hline CPB time (min) & $* 68.0 \pm 24.26$ & $64.45 \pm 26.24$ & 0.633 \\
Cross clamp time (min) & $* 27.0 \pm 10.08$ & $29.1 \pm 16.72$ & 0.763 \\
Total operation time (min) & $280.45 \pm 58.83$ & $295.47 \pm 96.25$ & 0.536 \\
Mechanical ventilation time (min) & $* 181.85 \pm 89.53$ & $354.63 \pm 218.46$ & 0.002 \\
Re-intubation & $\mathrm{Nil}$ & $\mathrm{Nil}$ & \\
Re-exploration & $\mathrm{Nil}$ & $\mathrm{Nil}$ & \\
Post operative ionotropes & $3(15 \%)$ & $47.29 \pm 13.3 \%)$ & 0.001 \\
ICU stay $($ hr) & $* 23.92 \pm 8.74$ & $\mathrm{Nil}$ & \\
Re-admission to ICU & $\mathrm{Nil}$ & $179.99 \pm 53.56$ & 0.001 \\
Post operative hospital stay $(\mathrm{hr})$ & $* 74.65 \pm 21.82$ & $\mathrm{Nil}$ & \\
Major Complications & $\mathrm{Nil}$ & $\mathrm{Nil}$ & \\
30 days mortality & $\mathrm{Nil}$ & & \\
\hline
\end{tabular}

*Mean $\pm \mathrm{SD}$

fast-track group (all 20 patients) which was 74.65 hours (3.1 days) and conventional group which was 179.99 hours (7.5 days). There was no major inhospital or out-of-hospital complications in either group. No patient was readmitted at our centre or elsewhere for any complication arising from the procedure. There was no mortality in either group.

\section{Discussion:}

Multiple studies from the Western world have shown the safety and efficacy of fast-track cardiac surgery. ${ }^{8}, 10,14-15$ It has conclusively been demonstrated that this approach does not compromise the quality of care. Rather it leads to early ambulation, increase patient and parent satisfaction, earlier return to school and less burden on the hospitals and health care providers. This facility is not available in our country and the reasons for this are multiple. 1) Cardiac surgical procedures are still performed mainly in Dhaka city and the non-cardiac doctors are reluctant to manage any medical / surgical condition in a cardiac patient. 2) Though it is well established that most patients with minimal co-morbid conditions and stable haemodynamics can be safely admitted on the day of surgery, ${ }^{16-17}$ here patients stays for many days preoperatively $(18 \pm 98$ days in this hospital), as they are admitted without complete workup, which is performed once the patient is in-hospital. 3) The poor educational level of patients / parents is another factor that causes a sense of insecurity if the patient is discharged early from the hospital. But our experience shows that, carefully selected low risk patients could be discharged early from the hospital without any adverse outcome. In the present study we could discharge about $90 \%$ of operated patients within 3 days of surgery without any adverse outcome and without compromising the quality of care. We discharged the patient only when there were no existing or anticipated adverse events; in case of the latter, the patients were not discharged till deemed to be fit. We believe that some of our practices might have contributed towards rapid recovery and early discharge from the hospital. Some of these include: normothermic cardiopulmonary bypass, maintenance of relatively high haematocrit, standardized surgical procedures, minimal use of blood products, short acting anesthetic/analgesic agents, ${ }^{3,4}$ full rewarming and maintaining normothermia, early extubation in the presence of a senior team member, early ambulation and intense physiotherapy, early resumption of enteral feeds, early removal of drains/invasive lines, judicious use of antibiotics and analgesics, emphasis on personal hygiene, and proper education of patients/parents. Besides these, a good communication between the operating team and the patients/parents is also essential.

\section{Conclusion:}

Fast tracking is feasible and safe in low-risk openheart surgery in children with cardio-pulmonary bypass in Bangladeshi scenario. A multidisciplinary 
approach with a set protocol is required to achieve this goal in a safe and reproducible manner. However, the hospital discharge should not be hastened, without proper evaluation of patient's condition, because it may lead to an increase in readmissions with a simultaneous decrease in patient safety and satisfaction.

\section{References:}

1. Kehlet H. Effect of postoperative pain treatment on outcome-current status and future strategies. Langenbecks Arch Surg 2004;389:244- 9.

2. Wilmore DW, Kehlet H. Management of patients in fast-track surgery. BMJ 2001;322:473- 6 .

3. Vidam V, Fosse E, Mollnes T, Garred P, Svennevig J. Complement activation with bubble and membrane oxygenators in aortocoronary bypass grafting. Ann Thor Surg 1990;50:387-91.

4. Gu Y, Wang Y, Chiang B, Gao X, Ye C, Wildeuver C. Membrane oxygenator prevents lung reperfusion in canine cardiopulmonary bypass. Ann Thor Surg 1991;51:573-8.

5. Royse C, Royse A, Soeding P. Routine immediate extubation after cardiac operation: A review of our first 100 patients. Ann Thorac Surg 1999;68:1326-9.

6. Neirotti RA, Jones D, Hackbarth R, Paxson Fosse G. Early extubation in congenital heart surgery. Heart Lung Circ 2002;11:157-61.

7. Meissner U, Scharf J, Dötsch J, Schroth M. Very early extubation after open-heart surgery in children does not influence cardiac function. Pediatr Cardiol 2008;29:317-20.

8. Fernandes AM, Mansur AJ, Caneo LF, Lourenco DD, Piccioni MA, Franchi SM, Afiune CM, Gadioli JW, Oliveira Sde A, Ramires JA. The reduction in hospital stay and costs in the care of patients with congenital heart diseases undergoing fast-track cardiac surgery.
Arquivos Brasileiros de Cardiologia 2004;83:27-34. 18-26.

9. Yanatori M, Tomita S, Miura Y, Ueno Y. Feasibility of the fast-track recovery program after cardiac surgery in Japan. Gen Thorac Cardiovasc Surg 2007;55:445-449

10. Howard F, Brown KL, Garside V, Walker I, Elliott MJ. 2009. Fast-track pediatric cardiac surgery: the feasability and benefits of a protocol for uncomplicated cases. Eur J Cardiothorac Surg 2010;37:193-196

11. Gooi J, Marasco S, Rowland M, Esmore D, Negri J, Pick A. Fast-track cardiac surgery: application in an Australian setting. Asian Cardiovasc Thorac Ann 2007; 15(2): 139-143.

12. Jenkins KJ, Gauvreau K, Newburger JW, Spray TL, Moller JH, Iezzoni LI. Consensus-based method for risk adjustment for surgery for congenital heart disease. $J$ Thorac Cardiovasc Surg 2002; 123: 110-118

13. Wernovsky G, Wypij D, Jonas RA, Mayer JE Jr, Hanley FL, Hickey PR, Walsh AZ, Chang AC, Castañeda AR, Newburger JW, Wessel DL. Postoperative course and hemodynamic profile after the arterial switch operation in neonates and infants. A comparison of low-flow cardiopulmonary bypass and circulatory arrest. Circulation 1995;92: 2226-2235.

14. Oxelbark S, Bengtsson L, Eggersen M, Kopp J, Pedersem J, Sanchez R. Fast track as a routine for open-heart surgery. Eur J Cardiothorac Surg 2001;19:460-3.

15. Vanek T, Brucek P, Straka Z. Fast track as a routine for open-heart surgery. Eur J Cardiothorac Surg 2002;21:369.

16. Arom KV, Emery RW, Petersen RJ, Schwartz M. Patient characteristics, safety, and benefits of same-day admission for coronary artery bypass grafting. Ann Thorac Surg 1996;61:1136-40.

17. Cella AS, Bush CA, Codignotto B. Same-day admission for cardiac surgery: a benefit to patient, family, and institution. J Cardiovasc Nurs 1993;7:14-29. 